\title{
Hemispatial asymmetries in judgment of stimulus size
}

\author{
Jennifer Charles, Arash Sahraie, and Peter McGeorge \\ University of Aberdeen, Aberdeen, Scotland
}

\begin{abstract}
Recent research has demonstrated a leftward bias in judgments of size. In the present experiments, hemispatial size bias was measured through simultaneous presentation of a circle and an ellipse varying in horizontal or vertical extent. A consistent leftward bias of horizontal size judgments (but not vertical) was obtained; at the point of subjective equality, the width of the objects that were presented in left hemispace was smaller than the width of the objects that were presented in right hemispace. These data suggest that the horizontal extent of stimuli appear larger in left hemispace than in right hemispace. Results also indicated that symmetrical stimulus presentation, with respect to the vertical meridian, is required for the bias to emerge. Furthermore, increasing or decreasing stimulus eccentricity weakened the effect. Attenuation of this bias upon the manipulation of parameters indicates that this phenomenon is context specific and is affected by similar parameters that are known to influence the magnitude of error in pseudoneglect.
\end{abstract}

Asymmetries in perception are frequently demonstrated in patients suffering from hemispatial neglect. Neglect patients typically exhibit a perceptual deficit to the left, following a lesion to the right parietal cortex. Patients suffering from unilateral hemispatial neglect fail to attend to or report stimuli that are presented in the visual field contralateral to their lesion, despite intact visual processing and visual acuity. Neglect can manifest itself in various forms, and consequently, patients are often unaware of one half of space (Rapcsak, Watson, \& Heilman, 1987) or of one side of an object (Driver \& Halligan, 1991). For instance, in horizontal line bisection tasks that are commonly used in the screening of neglect, patients tend to place their midline mark to the ipsilesional side of true center because of a failure to respond to-or the neglect of - the leftward extent.

A similar pattern of response, termed pseudoneglect (Bowers \& Heilman, 1980), has been demonstrated in normal observers. In this instance, however, observers tend to overestimate or over attend to objects presented to the left, resulting in comparative "neglect" of the right. Research suggests that this asymmetry is brought about by the dominance of the right hemisphere in mediating spatially directed attention (Foxe, McCourt, \& Javitt, 2003; Heilman, Bowers, Valenstein, \& Watson, 1987; Kinsbourne, 1970; Mattingley, Bradshaw, Nettleton, \& Bradshaw, 1994). The effect is not as marked as the asymmetry found in neglect patients; however, findings consistently reveal the tendency for normal observers to overestimate the leftward extent of a stimulus and to bias their response toward the leftward features. For instance, in line-bisection tasks, nonclinical participants tend to overestimate the leftward extent of a horizontal line (un- derestimating the length of the right side in comparison) and bisect the line slightly to the left of its true center (see, e.g., Jewell \& McCourt, 2000; Luh, 1995; McCourt \& Jewell, 1999; Milner, Brechmann, \& Pagliarni, 1992).

Reports of this phenomenon within normal observers have recently extended to judgments of stimulus luminosity, numerosity, and size (Nicholls, Bradshaw, \& Mattingley, 1999). Nicholls and colleagues used the grayscales task (see Mattingley et al., 1994), in which participants are asked to make a relative judgment of the brightness of two left-right mirror-reversed luminance gradients. Each luminance gradient (or "grayscale") was presented as a horizontal bar that changed incrementally from white on one side to black on the other. Two luminance gradients were presented on each trial, one above the display screen's center and the other below. Researchers have previously shown that when asked to choose which stimulus is darker overall, participants tend to choose the grayscale that is dark on the left-hand side, even though both stimuli are actually of equal luminosity (Mattingley et al., 1994). Similarly, Nicholls et al. (1999) demonstrated a leftward bias whereby participants tended to select the stimulus that contained the relevant feature on the left-hand side. This bias was found regardless of whether participants were asked to judge how bright or dark stimuli were. In addition, when the task was altered so that participants were asked to make judgments of numerosity or size, participants selected the stimulus in which features were more densely arranged - or that was larger - on the left-hand side. Furthermore, the authors demonstrated that this leftward bias persisted regardless of whether the task instructions were to select the stimulus containing more or less of a feature.

A. Sahraie, a.sahraie@abdn.ac.uk 
Some have suggested that these types of perceptual asymmetries found in normal observers are the result of an attentional or perceptual bias toward left hemispace (Luh, 1995; Mattingley et al., 1994) that is brought about by selective activation of the right hemisphere during spatial processing. For instance, activation of the right hemisphere during judgments of brightness, numerosity, or shape may generate a bias of attention to left hemispace, thereby increasing the salience of stimuli located there. As a result, when faced with stimuli that are essentially identical, participants bias their judgments toward stimuli containing more of the relevant feature on the left-hand side. Therefore, it is possible that a generalized right hemisphere specialization for attention leads to a bias of the left hemispace in normal observers. Certainly this hypothesis concurs with demonstrations that neglect patients with right hemisphere damage consistently show strong asymmetries in the opposite direction. Moreover, research has recently shown that right-handed participants judged stimuli that were presented to the left eye as being larger than those presented to the right eye, regardless of position within hemispace (McManus \& Tomlinson, 2004). Although the theoretical locus of this finding is unclear, the authors propose that it is possible that there are receptor differences between the two eyes, therefore, the effect may be similar to the attentional bias in stimulus judgment brought about through right-hemispheral dominance.

The tasks described above seem to involve similar cognitive mechanisms to those employed in line bisection tasks and appear to be measuring similar attentional biases. Moreover, recent studies have indicated that patients with hemispatial neglect actually perceive objects in right hemispace as being larger than physically identical objects in left hemispace (e.g., Milner \& Harvey, 1995). During a matching task, the authors found that neglect patients required horizontal bars that were presented on the left to be longer in order for them to appear equal in length to bars that were presented on the right, and for nonsense shapes to be larger on the left in order for them to appear of equal area to those that were presented in right hemispace. Interestingly, although presented as control data, normal observers demonstrated the opposite pattern of responses; they required objects on the right to be physically larger to look equal to those that were presented in left hemispace. Since this study involved the simultaneous presentation of two stimuli in different locations in space for comparison, it is notably different from previous research that has revealed objectbased left-right perceptual asymmetries within a stimulus (for instance, when the leftward extent of a horizontal line is overestimated and, consequently, misbisected).

If the type of size judgment task reported above elicits biases toward one side of space, then one can feasibly assume that a leftward bias may be found in normal observers in similar size estimation tasks. Therefore, one could predict that in addition to the object-based (withinstimulus) perceptual asymmetries previously identified, a leftward bias in normal observers may extend to the perception of objects presented in left and right hemispace.

The current study sought to examine perceptual asymmetries for objects that were presented in left and right hemispace. Sensitivity in detection of object size across hemispace was measured by presenting a circle simultaneously with an ellipse varying in horizontal or vertical dimension only. In one experimental manipulation, participants were asked to indicate which of the two objects was wider in horizontal direction, and in another, they were asked to report which object was taller in the vertical direction (see Figures 1A and 1B).

Recent reports summarized previously have indicated that asymmetries exist in the size perception of objects across hemispace in both neglect and control participants. Therefore, we predicted that observable differences should be obtained in the measurement of the perceived size of objects presented in the left and right hemispace in normal observers. Specifically, we predicted that participants would overestimate the size of objects that were presented in left hemispace in comparison with an object of equal size presented in right hemispace. Therefore, we anticipated that leftward biases would be obtained for judgments of both horizontal and vertical size.

\section{EXPERIMENT 1}

\section{Method}

Participants. Twelve undergraduate students from the University of Aberdeen participated in the experiment ( 7 female, 5 male) as
A

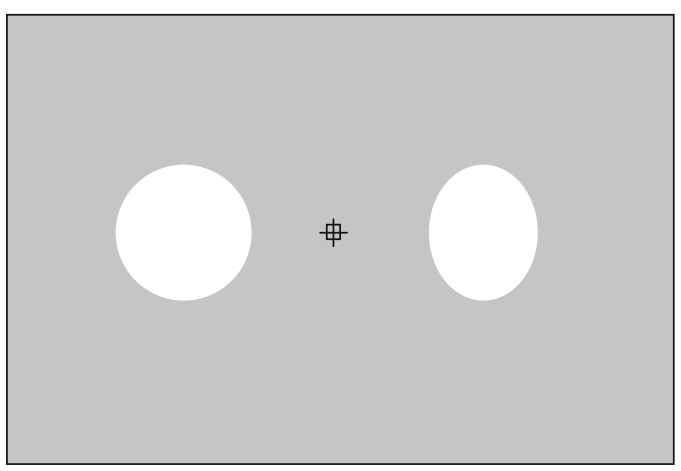

B

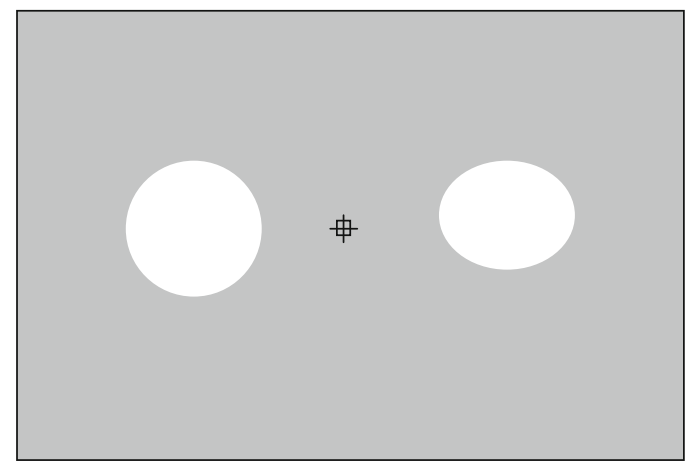

Figure 1. Experiment 1. (A) Horizontal judgment condition. Reference circle and ellipse varied by horizontal extent presented. (B) Vertical judgment condition. Reference circle and ellipse varied by vertical extent presented. 
part of their course requirements. Participants were 20-49 years old $(M=27)$. All participants were right handed (as determined by writing hand), and all had self-reported normal or corrected-to-normal visual acuity.

Apparatus. Stimuli were generated via a PC (Windows XP) using a specialized SVGA graphics card (VSG 2/5, Cambridge Research Systems, U.K.) and were displayed on a $21-$ in., 100-Hz monitor (Sony multiscan G520). The monitor was enclosed in a cubicle with a chinrest at a viewing distance of $640 \mathrm{~mm}$. Background luminance was set at $20 \mathrm{~cd} / \mathrm{m}^{2}$ and fixation was a high contrast black cross hair, subtending $0.5^{\circ}$. Participants responded with their right hand using a four-buttoned response box. Fixation accuracy was monitored throughout the experiment by using a modified ASL 5000 pupillometer (Applied Science Laboratories, MA).

Materials. The size estimation task consisted of one set of nine target ellipses varying in horizontal dimension only $\left(3^{\circ}\right.$ in height, width ranging through $2.5^{\circ}, 2.7^{\circ}, 2.8^{\circ}, 2.9^{\circ}, 3^{\circ}, 3.1^{\circ}, 3.2^{\circ}, 3.3^{\circ}$, and $3.5^{\circ}$ ) and another set of stimuli varying in vertical extent only (width $=3^{\circ}$, height ranging from $2.5^{\circ}$ to $3.5^{\circ}$ ). Each set of stimuli also contained a reference circle $\left(\right.$ diameter $\left.=3^{\circ}\right)$. On each trial, one target stimulus was presented to one side of the fixation, and the reference circle was presented on the other side of the fixation cross. Stimuli were presented along the horizontal meridian and centered at $5^{\circ}$ to the left or right of the central fixation point. In addition, stimulus eccentricities were systematically varied by adding a jitter of $\pm 0.5^{\circ}$ to the horizontal or vertical stimulus coordinates so that stimuli were not perfectly aligned in the display. Hence, participants could not rely on the distance from fixation or vernier acuity as cues to size judgment. The presentation of stimuli was counterbalanced so that each target ellipse was presented an equal number of times to the left and to the right side of fixation. Each block of trials contained 32 presentations of each target ellipse (and the reference), resulting in a total of 288 trials per block.

Procedure. During the task, a reference circle and an ellipse were presented at either side of a fixation cross along the horizontal meridian. Participants undertook two experimental conditions in which they were asked to indicate which object they thought was wider (horizontal judgment) or taller (vertical judgment). Participants completed two blocks of trials in succession (576 trials in total) in which they made horizontal size judgments, and two blocks (a further 576 trials) in which they made vertical size judgments. The order of experimental condition was counterbalanced across participants, and most participants undertook each condition in separate testing sessions (due to time constraints, in some cases, this was not possible). After each set of 30 trials, there was a short break, and participants restarted trials with a button press when they felt ready to continue. Each trial commenced with a high tone that was followed by an interval of $1,000 \mathrm{msec}$, after which, stimuli were presented for $100 \mathrm{msec}$. After stimulus presentation, participants were asked to respond as quickly and accurately as possible, indicating which object they thought was wider (or taller). As soon as the participant had made his or her response, the next trial began. Participants were not informed that in some of the trials, the dimensions of the two objects would actually be identical, but instead were told that they had to make a subjective judgment for each trial regardless of the objects' dimensions. Participants responded by pressing either the upper left or upper right key on the response box in order to select the object presented in left or right hemispace, respectively. Participants were given a set of eight practice trials before each experimental condition in order to familiarize themselves with the task.

\section{Results}

For each condition, participants' judgments of each size of target ellipse compared with the reference circle were recorded. Frequency data for each participant were obtained for horizontal and vertical size judgments of target ellipses as wider or taller than the reference circle. These responses were separated into judgments that were made when the reference circle was presented in left hemispace and judgments that were made when the reference circle was presented in right hemispace for comparison across hemispace. Probit analysis of frequency data yielded a psychometric function for each participant. From these functions, a point of subjective equality (PSE), in which target and reference were judged to be of equal size, was extracted from each observer, for each condition across hemispace. In each experimental condition, this extraction yielded 24 separate PSE values (for each participant, a PSE was obtained for targets that were presented in right hemispace and a PSE was obtained for targets that were presented in left hemispace, $n=12$ ). Paired-samples $t$ tests were then performed on the two distributions of 12 PSE values. Analyses of these data revealed a significant difference between size judgments of horizontal extent in left and right hemispace $[t(11)=2.32, p=.04]$, but not for judgments of vertical extent $[t(11)=1.36, p=$ .20]. For judgments of horizontal extent, the mean PSE for objects that were presented in left hemispace $\left(2.97^{\circ}\right)$ was significantly less than the PSE for objects that were presented in right hemispace $\left(3.04^{\circ}\right)$. These data are shown in Figure 2. Hence, stimuli that were presented in left hemispace were perceived to be wider than stimuli of equal size that were presented in right hemispace.

\section{Discussion}

The results revealed a consistent bias in horizontal size judgments of ellipses that were presented along the horizontal meridian. Target ellipses that were presented in left hemispace were judged to be wider than target ellipses that were presented in right hemispace; for horizontal orientation judgments, the point of subjective equality for target ellipses that were presented in left hemispace decreased, and the PSE for target ellipses that were presented in right hemispace increased. Thus, when two objects of an equal size were presented in left and right hemispace, ellipses that were presented to the left were judged to be longer in horizontal extent.

This finding suggests that in much the same way as normal observers misperceive true center in line-bisection tasks, there is an overestimation of the horizontal extent of

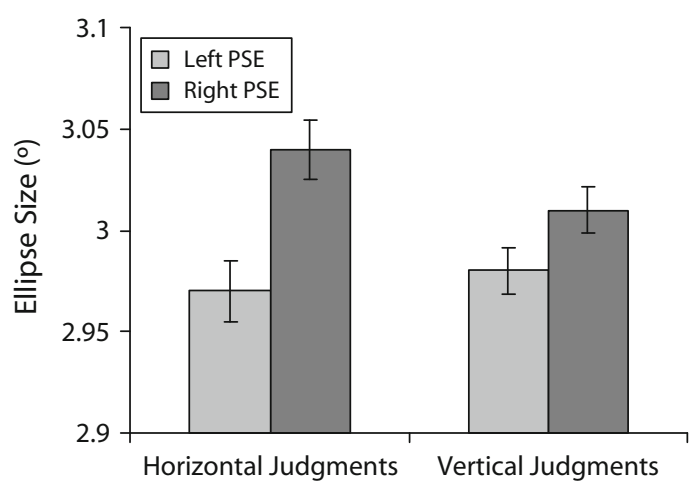

Figure 2. Mean PSE (point of subjective equality) values for stimuli that were presented in left and right hemispace across horizontal and vertical judgment conditions. 
objects that are presented in left hemispace, which results in a leftward bias for judgments of horizontal size. One possible explanation may be that the perception of space is distorted; left hemispace is enlarged in comparison with right hemispace; therefore, the left stimulus is overestimated when left and right stimuli are identical. However, the results of the vertical judgment condition suggest that this overestimation is specific to perception of horizontal size, since no leftward bias was found when participants were asked to judge the vertical extent of ellipses. Therefore, it appears that there is not a generalized expansion in the perception of left hemispace that would predict leftward biases for all orientations. Instead, when objects are presented along the horizontal meridian, the leftward size bias is specific to judgments of horizontal extent.

Recent studies manipulating stimulus orientation in line bisection tasks (McCourt \& Olafson, 1997) and grayscales tasks (Nicholls, Mattingley, Berberovic, Smith, $\&$ Bradshaw, 2004) have indicated that vertical and horizontal biases are moderated by independent attentional mechanisms. Given these findings, it seems unsurprising that differential effects were obtained for horizontal and vertical judgments in the present Experiment 1. The current data also concur with the results that Milner and Harvey (1995) obtained from normal observers performing a matching task in which they judged identical symmetrically presented horizontal rectangles to be longer on the left side than on the right side, and judged nonsense shapes to appear larger on the left side than on the right side. In contrast - and as predicted - neglect patients showed the opposite pattern of results; this group judged horizontal rectangles and nonsense shapes that were presented on the right to be longer and larger, respectively. Furthermore, in accordance with the present findings, no such bias was found for judgments of length of vertical rectangles presented on the left and right in normal observers or in neglect patients. In addition, using Milner and Harvey's matching task, Ferber and Karnath (2001) obtained differences between control and neglect patients in size judgments of horizontal rectangles that were displayed to the left and right side of visual space. The authors reported that neglect patients (and some patients with hemianopia) revealed a lateralized (to the left) underestimation of horizontal object size. No such deficit in horizontal size estimation was found when horizontal rectangles were arranged vertically one above the other, or more importantly, when the size of horizontally arranged vertical rectangles were judged. Therefore, the underestimation of the horizontal extent observed does not appear to be accounted for by a general underlying misperception of object size on the left side of visual space.

Since left-right spatial asymmetries in judgment were only obtained for horizontally oriented stimuli, Ferber and Karnath (2001) have suggested that the asymmetry in judgment of nonsense shapes can be attributed to the perceptual distortion of horizontal extent. Some have previously proposed that visuospatial neglect may involve a generalized shrinkage of size perception in the affected hemispace (see, e.g., Gainotti \& Tiacci, 1971; Milner, Harvey, Roberts, \& Forster, 1993). However, data from the matching tasks described above and the results of the present experiment indicate that there might be a perceptual distortion specific to horizontal size in healthy adults. It appears that in normal observers, the horizontal extent of stimuli is overestimated in left hemispace, which causes an asymmetry in judgment of horizontal size.

\section{EXPERIMENT 2}

Milner and Harvey's (1995) analysis of normal observers' error rates in horizontal and vertical judgment tasks ruled out the interpretation that their findings may be due to the vertical judgment task being more intrinsically difficult. However, the nature of the horizontal and vertical size judgment tasks in Experiment 1 may not be comparable because there is not equal spatial distribution of stimuli relative to the judgment required in each condition. For example, in the horizontal judgment condition, stimuli were distributed horizontally $\left(5^{\circ}\right.$ from the vertical meridian), and participants were asked to make judgments of horizontal orientation. In contrast, there was no vertical distribution of stimuli in the vertical judgment condition. Therefore, in Experiment 2, stimuli were presented asymmetrically (see Figure 3 ) so that there was equal horizontal and vertical distribution of stimuli, and judgments that were made about horizontal and vertical extent were comparable.

Judgments of object size were measured in the same way as that in Experiment 1-through the simultaneous presentation of a reference circle on one side and an ellipse varying in either horizontal or vertical extent on the other side of fixation. As they did before, participants made judgments of size in two experimental conditions, one in which they were asked to report which stimulus was wider (horizontal judgment) and another in which they were asked to report which stimulus was taller (vertical judgment). Given that Experiment 1 revealed a significant leftward bias for horizontal size judgments of ellipses, we predicted that a leftward bias would be obtained in horizontal size estimation in the present experiment. In addition, since horizontal and vertical distributions have been made comparable through asymmetrical stimulus presentation, we anticipated that a leftward vertical size judgment bias would be obtained.

\section{Method}

Participants. Fourteen undergraduate students (10 female, 4 male) at the University of Aberdeen volunteered to participate in this study. The participants' ages ranged from 19 to 56 years $(M=$ 24). All were right-handed (as determined by writing hand) and had self-reported normal or corrected-to-normal visual acuity.

Apparatus and Materials. Stimuli were generated and displayed using the same equipment as that in Experiment 1. The same procedure and number of trials were administered, and participants' responses were made and recorded in the same way. As before, the size estimation task consisted of one set of nine target ellipses varying in horizontal dimension only (height $=3^{\circ}$, width $=2.5^{\circ}-3.5^{\circ}$ ), another set of stimuli varying in vertical extent only (width $=3^{\circ}$, height $\left.=2.5^{\circ}-3.5^{\circ}\right)$, and a reference circle $\left(\right.$ diameter $\left.=3^{\circ}\right)$. On each trial, one target stimulus was presented on one side (left-right) of the fixation cross, and the reference circle was presented on the other. Stimuli were centered at a horizontal distance of $5^{\circ}$ from the cen- 
A

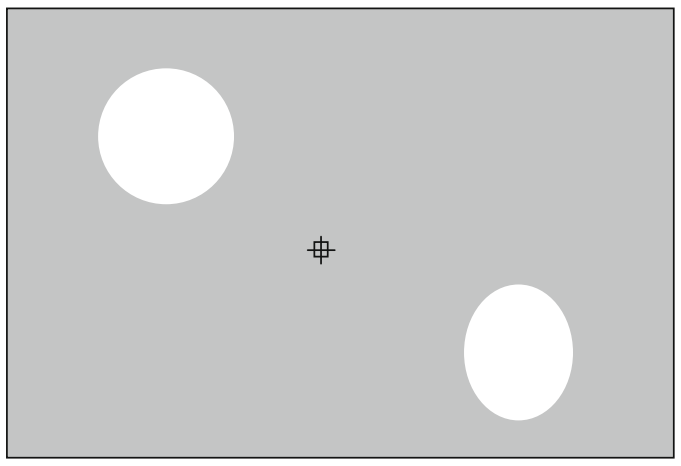

B

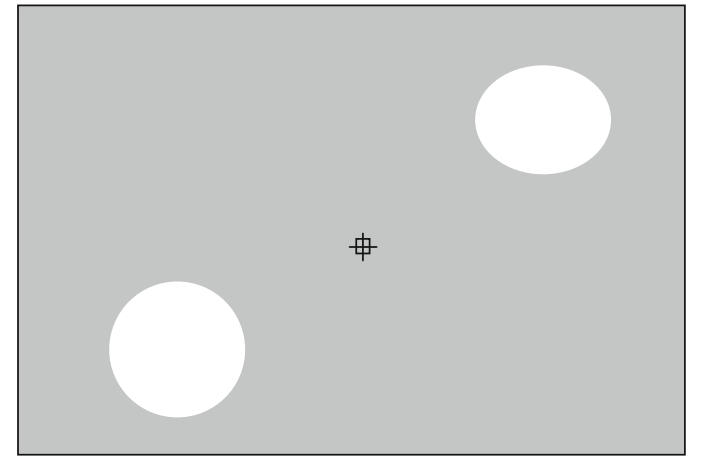

Figure 3. Experiment 2. Asymmetrical stimulus presentation. In the horizontal judgment condition (A), a circle and ellipse varied by horizontal dimension are presented. In the vertical judgment condition (B), a circle and ellipse varied by vertical dimension are presented. The diagrams show configurations used in Blocks 1 (A) and 2 (B), data from which are combined in each condition. The complimentary blocks include horizontal judgment when the left target is presented below the horizontal meridian and the right target above, and vertical judgment when the left target is presented above the horizontal meridian and the right target below.

tral fixation point at an eccentricity of $7.1^{\circ}$. However, stimuli were presented asymmetrically so that for each condition, in one block of trials, targets that were presented in left hemispace were located $5^{\circ}$ above the horizontal axis and targets that were presented in right hemispace were located $5^{\circ}$ below the horizontal axis, and vice versa for the second block. In each condition, stimulus eccentricities were systematically varied by $\pm 0.5^{\circ}$ in horizontal or vertical dimension so that participants could not use distance from fixation as a cue to size judgment.

Procedure. Participants undertook the same experimental procedure that was described in Experiment 1 . The only different aspect about the task was that in the present study, participants were asked to make size judgments about stimuli that were presented asymmetrically with respect to the vertical meridian. During the task a reference circle and an ellipse were presented (one in left, upperlower hemispace and the other in right, upper-lower hemispace). Again, participants undertook two experimental conditions in which they were asked to indicate which object they thought was wider (horizontal judgment) or taller (vertical judgment). As they did before, participants responded by pressing either the upper-left or upper-right response key to select the object presented in left or right hemispace, respectively.

\section{Results}

Data were analyzed as those before, and responses were separated into judgments that were made when targets were presented in left hemispace and judgments made when targets were presented in right hemispace for each condition. Paired-samples $t$ tests on PSE data revealed no significant differences between left and right hemispace for horizontal judgments $[t(13)=-0.83, p=.42$; left $\mathrm{PSE}=2.98$, right $\mathrm{PSE}=3.01]$ or vertical judgments $[t(13)=-1.12, p=.29$; left PSE $=2.97$, right PSE $=$ 3.02]. These data are shown in Figure 4. Although there is a trend in the data whereby targets in left hemispace are judged to be wider and taller than those presented in right hemispace, these differences are not statistically significant. Hence, in this experiment, there were no leftward biases for either vertical or horizontal size judgments of stimuli that were presented asymmetrically with respect to the vertical meridian.

\section{Analyses by Superior and Inferior Hemispace}

A number of studies have also reported a bias for superior hemispace in vertical line bisection performance (e.g., Drain \& Reuter-Lorenz, 1996; Neilson, Intriligator, \& Barton, 1999; Shelton, Bowers, \& Heilman, 1990; Toth $\&$ Kirk, 1996). In order to determine whether a superior bias was evident in the current experiment, data were analyzed separately in terms of size estimations that were made in upper and lower hemispace for both vertical and horizontal judgment tasks. Data for vertical judgments revealed no significant difference between judgments that were made in Block 1 [left upper and right lower presentations; upper PSE $=2.98$, lower PSE $=3.04 ; t(13)=$ $-0.87, p=.40$ ] or in Block 2 [right upper and left lower presentations; upper PSE $=3.01$, lower PSE $=2.96$; $t(13)=-1.21, p=.25]$. In addition, for horizontal judgments, there was no significant difference between superior and lower hemispace in Block 1 [upper PSE $=3.03$, lower PSE $=2.98 ; t(13)=1.19, p=.26$ ], and in Block 2 , significant differences were not in the predicted direction [upper PSE $=3.06$, lower PSE $=2.93 ; t(13)=-2.45$, $p=.03]$. Therefore, it seems unlikely that the lack of leftward bias in horizontal and vertical judgments can be explained by a competing bias toward superior hemispace.

\section{Discussion}

The results of the vertical size judgment condition revealed that there was no leftward vertical size bias for ellipses when they were presented asymmetrically with respect to the vertical meridian. This finding concurs with results that were presented by Milner and Harvey (1995) and Ferber and Karnath (2001), which reported no asymmetry for judgments of vertical rectangles that were presented in left and right hemispace. In addition, the findings from the current study replicate those of Experiment 1 in which no bias was found for vertical estimations of stimuli that were presented in left or right hemispace. These results again suggest that leftward perceptual biases 


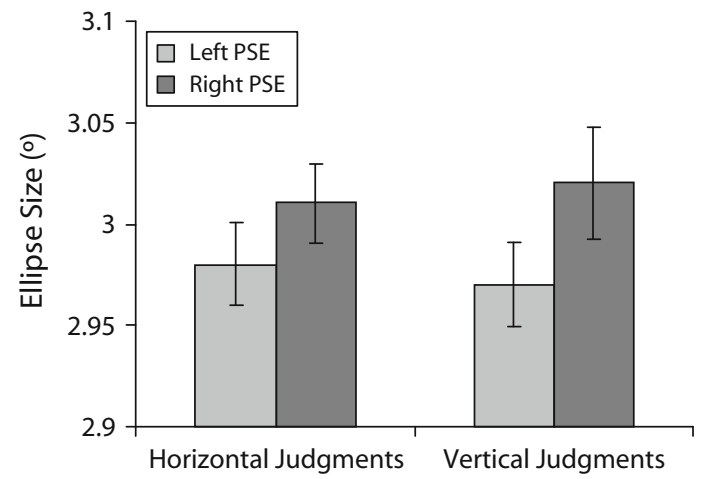

Figure 4. Mean point of subjective equality (PSE) values obtained in horizontal and vertical judgment conditions for asymmetrically arranged stimuli.

are specific to particular judgments and are not observed in size evaluations of all orientations.

Unexpectedly, however, the leftward bias that was obtained for horizontal judgments in Experiment 1 is not evident in the current experiment involving stimulus presentation that is asymmetrical with respect to the vertical meridian. Moreover, results of separate analyses by block revealed that these results are not mediated by biases for upper hemispace. However, these data might testify to the role of stimulus symmetry in facilitating the computation of object size. Indeed, some have posited that symmetry can be detected effortlessly - or even preattentivelyoften bypassing attentional resources that are usually required for judgment tasks (see Locher \& Wagemans, 1993; Wilkinson \& Halligan, 2002). Therefore, it appears that implicit cues to perceptual judgment inherent in symmetrically presented stimuli are absent in oblique presentation of stimuli and, as a result, the leftward horizontal bias is attenuated. Recent investigation has shown that stimulus symmetry can facilitate bisection of shapes and that symmetrical shapes are identified with greater accuracy than asymmetrical shapes (Wilkinson \& Halligan, 2003). Hence, removal of potentially useful symmetry cues may cause a drop in task performance and negate the effects of perceptual bias in the current study.

\section{EXPERIMENT 3}

Experiment 1 demonstrated a leftward bias in the perception of the horizontal extent of ellipses presented in hemispace. When asked to judge the width of ellipses, participants systematically overestimated the horizontal extent of ellipses presented in left hemispace. The data thus far have also indicated that this bias does not extend to judgments of vertical extent in conditions in which stimuli are presented symmetrically (in Experiment 1) or asymmetrically (in Experiment 2). Therefore, in the tasks to follow, participants were asked to make judgments of horizontal extent only.

No leftward horizontal size bias was obtained in Experiment 2 using asymmetrical stimulus presentation. This result suggested that symmetrical stimulus presentation (with respect to the vertical meridian) may be necessary for operation of a leftward horizontal size bias, potentially facilitating judgments through the elicitation of automatic cues. It is well documented that such symmetry is important in the judgment of stimuli and that vertical symmetry is easily detected (Barlow \& Reeves, 1979; Wenderoth, 1994), even when occurring at low levels among complex biological images (Evans, Wenderoth, \& Cheng, 2000). It is thought that the activation of a mirror stimulus representation strengthens responses to stimuli, and indeed, some researchers have suggested that symmetry perception occurs as a consequence of low-level grouping processes that operate automatically across all input (see Rainville \& Kingdom, 2000). In addition, evolutionary accounts of symmetry detection indicate that we are in some way predisposed to pick up symmetry cues (see Giurfa, Eichmann, \& Menzel, 1996; Moller \& Thornhill, 1998). In the present research, leftward biases have been obtained for stimuli that were presented symmetrically across the vertical axis in left and right hemispace and along the horizontal meridian. Therefore, it is unclear whether it is symmetry per se or symmetry specifically about the horizontal meridian that is required to produce the horizontal size bias. To address this issue, Experiment 3 employed symmetrical stimulus presentation in two experimental conditions in which stimuli were presented along the horizontal meridian or in upper hemispace ( $5^{\circ}$ above the horizontal meridian). Participants made horizontal judgments of objects that were presented in each of these conditions (see Figure 5).

We hypothesized that a leftward bias would be obtained for stimuli that were presented along the horizontal meridian, replicating the horizontal judgment bias that was found in Experiment 1. In addition, given the importance of vertical symmetry in object processing, we predicted that a leftward bias would also be obtained for stimuli that were presented symmetrically in upper hemispace. However, because more attention is required to process stimuli further into the visual field (see DeValois \& DeValois, 1988), we predicted that increased eccentricity of stimulus presentation from fixation in this task would reduce the magnitude of leftward bias obtained.

\section{Method}

Participants. Sixteen students ( 9 female, 7 male) at the University of Aberdeen either volunteered to participate in this study or took part in return for course credit. The participants' ages ranged from 18 to 29 years $(M=24)$. All were right-handed (as determined by writing hand) and had self-reported normal or corrected-tonormal visual acuity.

Materials and Procedure. Stimuli were generated and displayed as those in the previous experiments and, unless stated otherwise, the experimental procedure was the same. However, the size estimation task consisted of a set of nine target ellipses varying in horizontal dimension only (vertical height $=3^{\circ}$, horizontal width ranging from $2.5^{\circ}$ to $\left.3.5^{\circ}\right)$ and a reference circle $\left(3^{\circ} \times 3^{\circ}\right)$. In two separate experimental conditions, participants made horizontal size judgments about stimuli presented along the horizontal meridian or in upper hemispace. In the condition in which objects were presented along the horizontal meridian, stimuli were centered at a horizontal distance of $5^{\circ}$ from the central fixation point. In the upper hemispace presentation condition, stimuli were centered $5^{\circ}$ to either side of the fixation along the horizontal meridian and $5^{\circ}$ above the horizontal axis. 
A

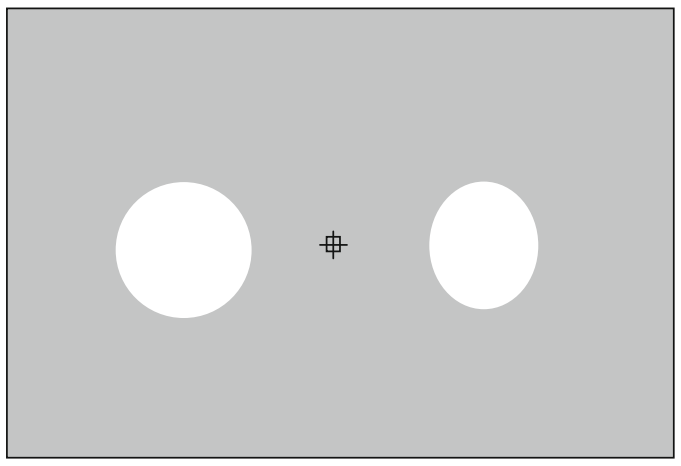

B

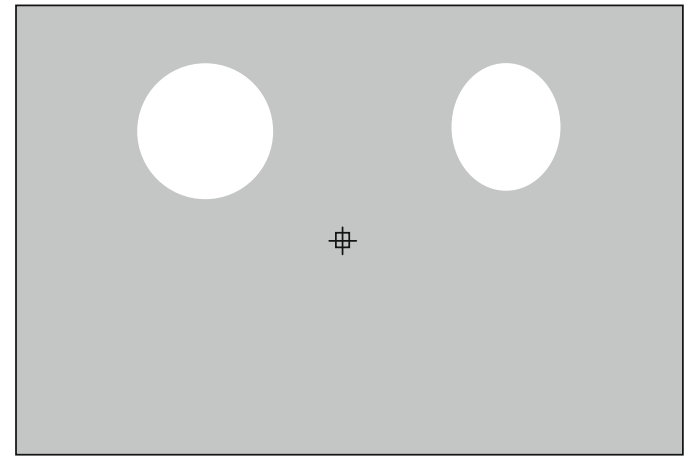

Figure 5. Experiment 3. Reference circle and ellipse varied by horizontal extent presented (A) along the horizontal meridian and $(B)$ in upper hemispace.

\section{Results}

Data were analyzed as those before, and comparisons were made for judgments in each condition across left and right hemispace. Paired $t$ tests revealed a significant leftward bias for judgments of ellipses presented along the horizontal meridian $[t(15)=-2.46, p=.03$; leftward $\mathrm{PSE}=2.98$, rightward PSE $=3.03]$ and for ellipses presented symmetrically in upper hemispace $[t(15)=-2.02$, $p=.03$ (one-tailed); leftward PSE $=2.98$, rightward $\mathrm{PSE}=3.01]$. These data are illustrated in Figure 6 .

\section{Discussion}

The results of Experiment 3 have primarily replicated the findings that were obtained in Experiment 1, revealing a leftward overestimation of the horizontal size of ellipses presented symmetrically along the horizontal meridian. In addition, the data reveal a significant leftward bias for stimuli presented symmetrically in upper hemispace. However, as was predicted, the magnitude of this bias is reduced compared with the size of effect obtained in presentation along the horizontal meridian. Therefore, it seems that increased eccentricity of stimuli from fixation when presented in upper hemispace attenuates the hemispatial bias. Interestingly, however, larger leftward errors have been found on the bisection of horizontal lines presented in the upper versus the lower visual field (McCourt \& Garlinghouse, 2000a; McCourt \& Jewell, 1999). On the basis of their findings, the authors propose that this effect is retinotopic in nature and suggest that the leftward bias in spatial attention across visual field elevation varies systematically, with stronger biases found in the superior hemifield and weaker effects found in the lower hemifield. Therefore, it would be of interest to examine whether a similar leftward bias would be obtained in the current task if stimuli were presented at the same eccentricity in the lower hemifield.

Space-based models of visual attention show that visual selection is biased toward favoring stimuli positioned near fixation over stimuli located far from fixation. One assumes that selection arises because of the increase in attentional weight that is attached to stimuli located near to fixation (see LaBerge \& Brown, 1989). For instance, in visual search tasks, targets at central locations are detected more quickly than targets at peripheral locations, even when the display durations that are employed are too brief to allow for eye movements (Carrasco, Evert, Chang, \& Katz, 1995). In addition, although eccentricity-dependent resolution losses are sometimes compensated for in psychophysical experiments by magnifying (scaling) stimuli at each eccentricity, advantages for central stimuli are still found on peripheral target trials if distractors that share response-relevant features fall in more central regions (Wolfe, O'Neill, \& Bennet, 1998). Therefore, in the current task, it seems that the advantages afforded to central stimuli-in addition to symmetrical cues to facilitate responses - increase leftward spatial biases (potentially through simplification of the task). Although there is evidence of a leftward size overestimation of stimuli presented in upper hemispace, the effect is attenuated in comparison with judgments made about stimuli presented along the horizontal meridian. In Experiment 4, investigation of the effects of stimulus eccentricity was extended, specifically to examine the nature of horizontal biases when eccentricity is varied along the horizontal meridian. In this experiment, stimuli were presented along the

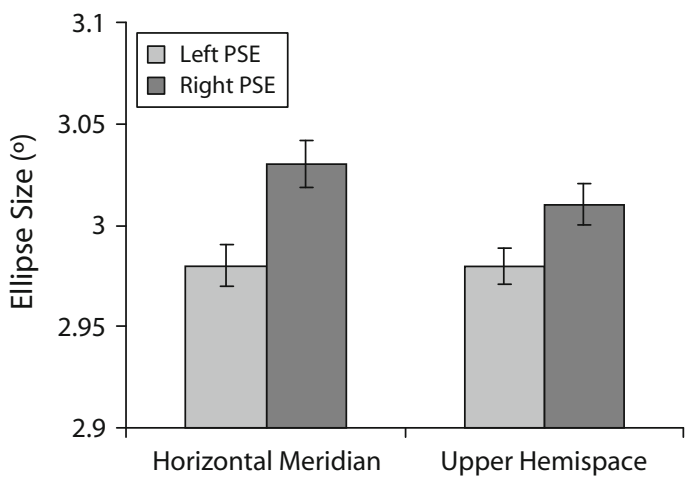

Figure 6. Mean point of subjective equality (PSE) values for horizontal size judgments of stimuli presented along the horizontal meridian and in upper hemispace. 
horizontal meridian, and stimulus eccentricity was manipulated across three conditions.

\section{EXPERIMENT 4}

The previous experiments have reported a reliable leftward overestimation of horizontal size for ellipses presented along the horizontal meridian. An additional, but relatively weaker, asymmetry was obtained for judgments of stimuli presented in upper hemispace. We proposed that despite symmetrical presentation, stimulus distance from fixation increases task demands and attenuates hemispatial bias.

In comparison, the present data suggest that the leftward horizontal size bias for stimuli presented along the horizontal meridian is relatively robust. Because results have indicated that this effect is strong, it was of interest to examine whether leftward bias for judgments of stimuli along the horizontal meridian was influenced by varying stimulus eccentricity. The objective of Experiment 4, therefore, was to examine the effects of varying stimulus eccentricity along the horizontal meridian. Stimulus eccentricity was manipulated across the horizontal meridian in three experimental conditions in which objects were presented at $2.5^{\circ}, 5^{\circ}$, and $10^{\circ}$ from fixation (see Figure 7 ). Participants made horizontal judgments about stimuli presented in each of the three conditions.

Two different theoretical models concerning the distribution of spatial attention in neglect have recently been proposed. Kinsbourne's activation orientation model (1987, 1993) proposes that each hemisphere controls a vector of attention increasing in strength from ipsilateral space to contralateral space. Following this model, one could argue that the magnitude of hemispatial bias is determined by the distance between stimuli in each trial, and that biases are accentuated at configurations in which objects are furthest apart and are minimized at locations where stimuli are close together. Indeed, researchers have shown that increasing deficits in neglect patients exist toward contralateral extremities (De Renzi, Gentilini, Faglioni, \& Barbieri, 1989). For instance, a gradient of attention has been revealed in line bisection tasks. As previously indicated, the current tasks appear to engage similar processes as do line bisection tasks and hence may be affected in comparable ways. Factors such as line length have been shown to markedly affect bisection errors in neglect patients and in normal observers, revealing a paradoxical "crossover" effect that occurs when shorter line lengths are used as stimuli (see Jewell \& McCourt, 2000). In healthy observers, shorter lines are usually bisected to the right of center, or reveal no bias at all (see, e.g., McCourt \& Jewell, 1999). Therefore, if the current task is similar to line bisection, then no bias should be observed when stimuli are located at closer eccentricities. Stimulus size - and in this task, we predicted eccentricity - is therefore important; the use of short lines reverses the direction of effects obtained with longer line lengths, and a number of studies have also revealed an increase in the magnitude of leftward bias as a function of line length (e.g., Halligan, Manning, \& Marshall, 1991; Luh, 1995; Manning, Halligan, \& Marshall, 1990). Again, one may assume that larger perceptual asymmetries occur with stimuli of longer lengths, because left and right stimulus extremities fall into areas of increased attentional asymmetry. This assumption - and the paradoxical effect of using shorter stimuli-suggests that hemispatial bias should increase with increased stimulus eccentricity from fixation. Therefore, one could predict that leftward bias for horizontal size will increase as stimuli are presented further into the visual field.

Alternatively, on studying line bisection performance in neglect, Anderson (1996) has proposed a computational model of spatial attention. According to this model, the spatial distribution of attention is determined by the salience of each point across left-right extrapersonal space. The model is based on the assumption that the right hemisphere attentional system attends to space bilaterally, whereas the left hemisphere system attends primar-

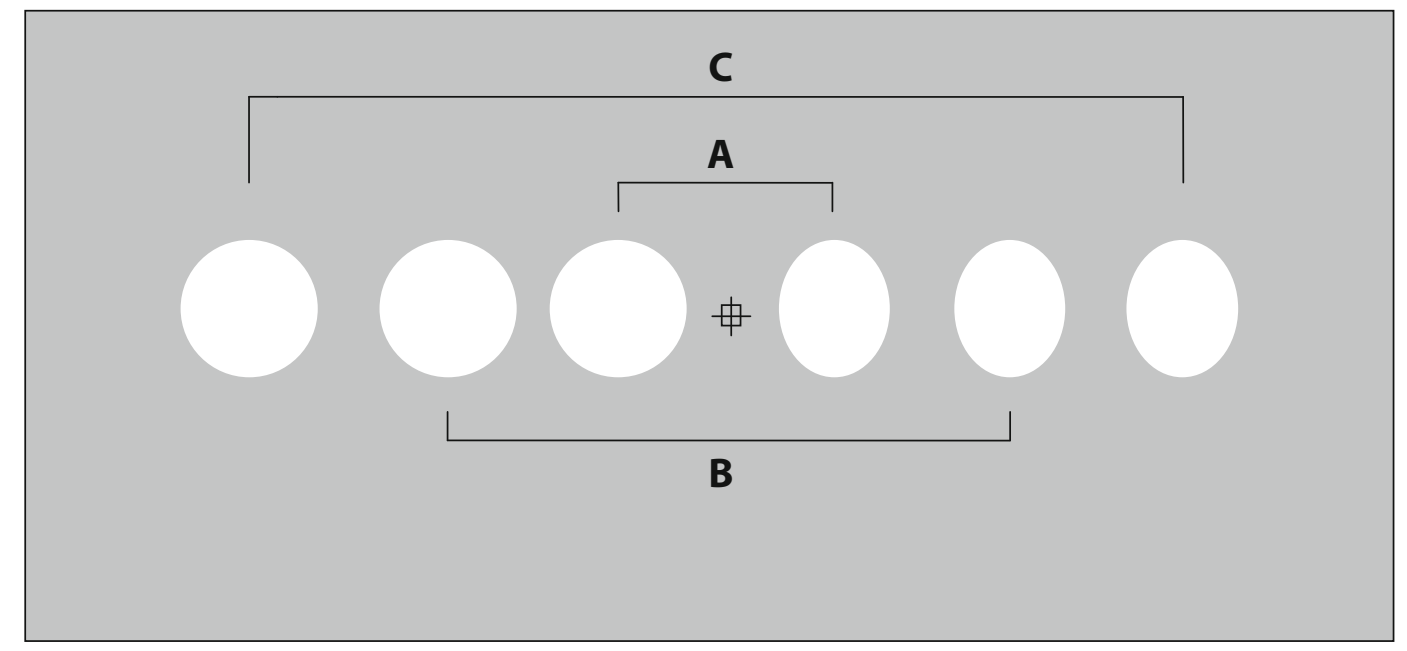

Figure 7. Experiment 4, horizontal judgments. Stimulus eccentricity varied along the horizontal meridian. Reference circle and ellipses presented (A) $2.5^{\circ}$, (B) $5^{\circ}$, and (C) $10^{\circ}$ from fixation. 
ily to right hemispace (Heilman \& Van Den Abell, 1980) and proposes that the determination of the salience of each point is therefore the sum of the salience of the two hemispheral systems. The resultant bimodal distribution indicates the total salience of each point across horizontal space, such that the absolute magnitude of attention varies with increased eccentricity. Therefore, an alternative hypothesis made on the basis of this model is that leftward bias will decrease as a function of increased stimulus eccentricity.

\section{Method}

Participants. Thirteen undergraduate students ( 7 female, 6 male) at the University of Aberdeen volunteered as participants in this study. The participants' ages ranged from 18 to 24 years $(M=$ 20). All were right-handed, as assessed by a modified version of the Waterloo handedness questionnaire (Steenhuis \& Bryden, 1989; mean handedness score $=+26$ ), and had self-reported normal or corrected-to-normal visual acuity.

Materials and Procedure. Apparatus set up was identical to that of the previous experiments, with the exception that fixation accuracy was not monitored in the present study. Stimuli were generated and displayed as they were previously and the experimental procedure was also the same. Since only horizontal judgments were required, the same set of stimuli was used as that described in Experiment 3. Participants made horizontal size judgments in three separate experimental conditions. All stimuli were presented along the horizontal meridian, but were differentiable in terms of eccentricity from fixation. In the three experimental conditions, stimuli were presented at eccentricities of $2.5^{\circ}, 5^{\circ}$, and $10^{\circ}$. In order to ensure that participants could not use distance from fixation as a cue to size judgment, stimulus eccentricities were systematically varied along the horizontal by $\pm 0.50^{\circ}$ in the $5^{\circ}$ and $10^{\circ}$ conditions, and by $\pm 0.25^{\circ}$ in the $2.5^{\circ}$ condition.

\section{Results}

Data were obtained and analyzed as those previously, and the results are shown in Figure 8. Paired-samples $t$ tests revealed a significant leftward bias for judgments of stimuli presented in the $5^{\circ}$ condition $[t(12)=-2.85$; $p=.02$; leftward PSE $=2.96$, rightward $\mathrm{PSE}=3.05]$. Although responses reveal a trend toward leftward bias, there were no significant differences between judgments made in left and right hemispace in the $2.5^{\circ}[t(12)=$ $-0.17, p=.87$; leftward PSE $=3.00$, rightward PSE $=$ $3.01]$ or $10^{\circ}$ condition $[t(12)=-0.21, p=.84$; leftward $\mathrm{PSE}=3.00$, rightward PSE $=3.01]$.

\section{Discussion}

The data obtained in Experiment 4 reveal a significant leftward bias for horizontal size judgments of ellipses presented in the $5^{\circ}$ condition. This result replicates the effect that was previously obtained in Experiments 1 and 3 for stimuli that were presented $5^{\circ}$ from fixation. In contrast, no bias was obtained for judgments of stimuli presented at $2.5^{\circ}$ eccentricity or $10^{\circ}$ eccentricity. The result of the $10^{\circ}$ condition contradicts hypotheses proposing an increase in perceptual asymmetry with increased distance between stimuli in left and right hemispace (see, e.g., Kinsbourne, 1987, 1993). In addition, an increase in stimulus eccentricity along the horizontal plane in this size estimation task seems to affect responses differently than increasing

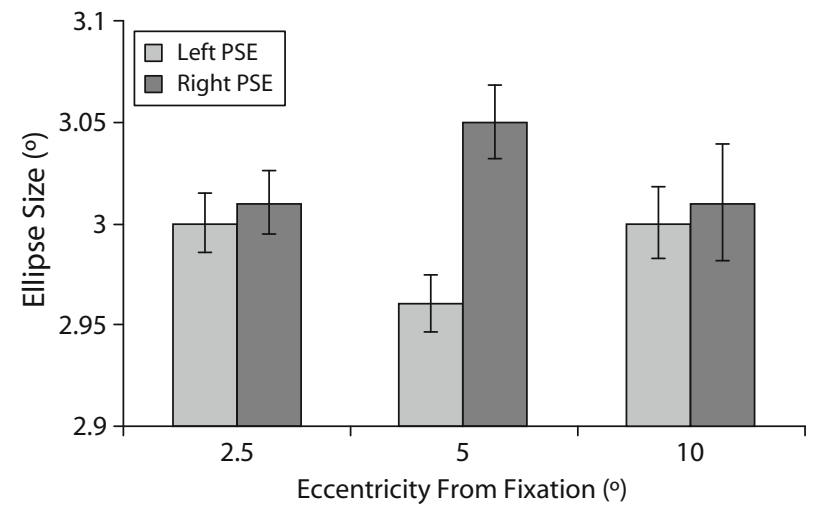

Figure 8. Mean point of subjective equality (PSE) values for horizontal size judgments of stimuli presented at $2.5^{\circ}, 5^{\circ}$, and $10^{\circ}$ eccentricity.

stimulus length in line bisection tasks, where increased bias has been reported.

However, this finding does concur with the prediction arising from Anderson's (1996) model that leftward bias would decrease with increased eccentricity (i.e., in the $10^{\circ}$ condition). In addition, a recent comparison of the two models outlined previously found that Anderson's salience model more accurately characterized the distribution of attention following right-sided damage in patients without hemispatial neglect, as indexed by fixations in a visual search task (Mapstone et al., 2003).

Moreover, the lack of bias revealed in the $10^{\circ}$ condition also concurs with the attenuation of bias that was revealed in Experiment 3 when stimuli were presented in left and right upper hemifields. Although a significant bias was obtained for stimuli that were presented in upper hemispace, horizontal eccentricity in this condition was maintained at $5^{\circ}$ from fixation, whereas in the $10^{\circ}$ condition, this distance was doubled. As was previously discussed, these data suggest that increased eccentricity from fixation serves to weaken hemispatial bias in judgments of horizontal size. Furthermore, the present experiment indicates that increased eccentricity along the horizontal plane in particular attenuates hemispatial bias. One explanation of this result is that because participants' attentional systems are aligned to the horizontal axis for making judgments of horizontal size in this task, observers may be especially sensitive to any change in stimulus parameters along the horizontal meridian. Thus, manipulations of this type may be more likely to affect responses. Certainly, the repeated bias currently found for symmetrical stimuli at horizontal eccentricities of $5^{\circ}$ suggests that the effect at this eccentricity is robust, and the attenuation of bias in other task conditions reveals that the observed asymmetry may be a result of interplay between a number of mechanisms, such as stimulus size and area of visual field in which objects are presented. For instance, following this line of thought, one might predict that scaling stimuli-in this case, enlarging stimuli that are presented at increased eccentricity — would simplify task demands (through cortical magnification) and, consequently, a leftward bias would emerge. 
The notion that responses are particularly sensitive to changes in stimuli along the horizontal meridian could also explain why no hemispatial bias was obtained when stimuli were presented in the $2.5^{\circ}$ eccentricity condition. Because the eccentricity effect commonly found would predict that stimuli at these locations are processed more quickly and accurately, this finding indicates that the leftward biases obtained in the $5^{\circ}$ condition are not simply due to the relative ease of processing of stimuli, since this premise would predict an increase in bias for judgments in the $2.5^{\circ}$ condition. Alternatively, although results in the $10^{\circ}$ eccentricity condition did not parallel line bisection data showing that attentional asymmetries are accentuated when longer stimulus lengths are employed, it could be that a similar counteractive effect of employing short line lengths in horizontal line bisection tasks occurs in size judgments of stimuli at locations close to fixation. In addition, this finding corresponds with the attentional gradient hypothesis that perceptual asymmetries will attenuate since stimuli are located nearer to the central periphery (see, e.g., Kinsbourne, 1987).

However, the horizontal distribution of spatial attention that was proposed by Anderson (1996) may also correspond with the lack of bias obtained in the $2.5^{\circ}$ condition. In Anderson's model, peak salience is shifted slightly leftward in the normal left-right spatial distribution. Therefore, any stimulus presented slightly to the left of visual space may be afforded more salience and could be overestimated. However, if stimuli are presented in left and right hemispace - but at locations close to fixation - stimuli might no longer fall on a salient point on the distribution curve, and the effect of leftward bias effects may not be of sufficient strength to emerge at this eccentricity. The slight leftward shift of peak salience proposed in the model suggests that bias across hemispace is specific and that effects might not be obtained for stimuli presented outside peak salience distributions.

Another factor that may impinge upon judgments of stimuli that are presented at close eccentricities may be the presence of a central spatial bias, recently demonstrated by Orr and Nicholls (2005). The authors used a modified version of the grayscales task in which luminance gradients were variously placed around the midline in participants' left or right hemispaces in order to explore perceptive bias in relation to the location of stimuli in the visual field. Stimulus orientation was manipulated so that object- and space-based coordinates were either congruent or incongruent. Even though a leftward object bias was found, results indicated that this result was moderated by overall bias for stimuli that were located more centrally. Therefore, a general preference for, or over attention to, centralized stimuli in the current judgment tasks may override any effect of left and right hemispace in size judgments made.

\section{GENERAL DISCUSSION}

Overestimation of the leftward extent of horizontal lines has frequently been observed in healthy participants in line bisection tasks, and more recently in tasks involving estimation of numerosity, luminosity, and size. These ex- periments have revealed biases toward leftward parts of an object upon judgment of a critical feature. What these tasks have in common, therefore, is that the biases revealed are object based to the extent that judgments are made about stimuli upon evaluation of left and right sides of individual objects. The present set of experiments aimed to examine whether leftward biases found in normal observers extend to the perception of objects in left and right hemispace. Through simultaneous presentation of two objects, one to each hemispace, we were able to investigate whether asymmetries exist in perception of the size of stimuli in space.

The present data have revealed a tendency for participants to systematically overestimate the horizontal extent of objects that are presented in left hemispace, upon utilization of particular stimulus parameters. No such bias was obtained for judgments of vertical size of objects in left and right hemispace. These results concur with previous findings that have reported left hemispace biases for size judgments of horizontal rectangles and nonsense shapes, but not for judgments of length of vertical rectangles (Ferber \& Karnath, 2001; Milner \& Harvey, 1995). Although this leftward bias does not extend to judgments of vertical extent of objects presented in left and right hemispace, an upward bias for vertical stimuli positioned in superior hemispace has been reported (Drain \& ReuterLorenz, 1996). Nicholls et al. (2004) have suggested that framing responses within certain decision contexts (left-right vs. up-down) may discourage the use of an orthogonal system. It could be that the alignment of the attentional system to the horizontal plane for response to horizontally presented stimuli conflicts with the vertically oriented judgment required during the task. Therefore, activation of a vertical attentional system (through up-down responses to vertically arranged stimuli) may be required for a bias of vertical extent to emerge.

Use of alternative strategies in judgment of stimuli has also recently been shown to influence the magnitude of leftward bias found in tasks (Nicholls, Mattingley, \& Bradshaw, 2005). Differences were found between the strength of bias in grayscales tasks when a global strategy was used or when stimuli were separated into components (halves or quarters) so that a comparative strategy was used. Leftward biases were obtained using both strategies; however, the magnitude of bias was reduced using the comparison strategy. The authors proposed that the global strategy may lend itself more to a space-based reference frame, whereas the comparison strategy may employ an object-based approach. Although research suggests that both reference systems contribute toward neglect and pseudoneglect (Nicholls et al., 2004; Post, Caufield, \& Welch, 2001; Tipper \& Behrmann, 1996), Nicholls et al. (2004) suggest that space-based mechanisms may give rise to stronger effects. Here, we highlight the context-specific nature of the attentional asymmetry and its sensitivity to changes in parameters. Furthermore, the amenability of these biases to changes in judgment strategy demonstrates the similarities in underlying cognitive processes in neglect and pseudoneglect.

It is generally accepted that pseudoneglect is due to a leftward attentional bias (see Halligan \& Marshall, 1994; 
Nicholls \& Roberts, 2002) that is brought about by the specialization of the right hemisphere for spatial tasks. However, the mechanism by which attention is distributed between left and right visual space in these tasks has not been determined. Researchers have suggested that the bias that is revealed in neglect patients is attributed to a generalized compression or shrinkage in size perception of the affected half of space (Gainotti \& Tiacci, 1971; Milner et al., 1993). Conversely, then, one could propose that pseudoneglect involves an overestimation of the leftward features of stimuli, or in this instance, in size perception of left hemispace. Yet, the results of the current experiments and those described previously suggest that this leftward overestimation is not a generalized distortion of object size in left hemispace. The data seem to be specific to judgments of horizontal extent and suggest that there is a miscomputation or overestimation of horizontal size in leftward space. Furthermore, this bias does not appear to reflect a general deficit in the estimation of horizontal size, since typically no differences are observed in error rates for vertical and horizontal judgments (Milner \& Harvey, 1995), and no differences have been found between patients and healthy observers when judging the horizontal size of vertically displayed rectangles (Ferber \& Karnath, 2001). In addition, Milner and Harvey attributed the underestimation of the area of nonsense shapes by neglect patients in their study to a specific distortion of horizontal extent that subsequently influences judgments of overall size.

The current studies add to an increasing body of research that has revealed leftward horizontal biases among normal observers (see, e.g., Ferber \& Karnath, 2001; Luh, Rueckert, \& Levy, 1991; McCourt, Freeman, TahmahkeraStevens, \& Chaussee, 2001; McCourt \& Garlinghouse, 2000b; Milner \& Harvey, 1995; Nicholls et al., 1999; Nicholls et al., 2004; Post, Caufield, \& Welch, 2001). The replication of leftward bias effects found at $5^{\circ}$ eccentricity and the attenuation of bias with manipulation of parameters testify to the specificity of the effect and suggest that the asymmetry does not generalize to all areas of left and right visual space. Indeed, the data seem to concur with Anderson's (1996) model of spatial attention, which suggests that the magnitude of attention (and, hence, magnitude of bias) diminishes with eccentricity and, moreover, specifies particular areas of left and right visual space that may be more susceptible to leftward bias effects. Visual selection theories have also accounted for an absence of bias at increased stimulus eccentricities, which suggests that the increase in attentional resources that are required to process stimuli at far eccentricities accounts for poorer performance in response to these stimuli. Since the leftward bias appears to rely on symmetry for its operation, one may presume that decreasing task demands serves to increase the magnitude of bias found. For instance, investigation of the effects of scaling stimuli to eccentricities would be of interest and, indeed, it may be that a leftward bias would reemerge upon cortical magnification of stimuli at increased eccentricities.

Experiment 3 demonstrated the importance of symmetrical stimulus presentation in the production of leftward bias. Asymmetries between judgments in left and right hemispace were obtained even when stimuli were presented symmetrically above the horizontal meridian. The current studies have revealed that leftward biases that were previously obtained for object-based judgments do extend to judgments of stimuli that are presented in left and right hemispace, but have further implicated symmetry and specificity of stimulus parameters in mediation of the space-based effect. The elimination of bias in judgment of stimuli close to fixation also reiterates the specificity of the asymmetry and demonstrates a finding similar to the attenuation of effect obtained in the line bisection literature using short line lengths. However, note that although the current task is similar to horizontal line bisection, the nature of stimulus presentation differs between the tasks. In line bisection tasks, only one stimulus is presented, and the asymmetry observed results as a consequence of judgment about one continuous stimulus over its entire area. Conversely, in the present size judgment task, stimuli were presented in discrete locations in left and right hemifields. Therefore, one way in which disparity may arise between the two tasks could be due to the difference between asymmetries arising from continuous versus discrete stimuli.

The task that was presently employed appears to produce effects that are variable and specific to the use of certain parameters, such as symmetrical stimuli and eccentricity. Parameters such as displacement and line length have similarly been shown to influence the magnitude of error in neglect patients. Indeed, the context-specific nature of the current bias is in accordance with the variability of results described in the line bisection and neglect literature. Therefore, if the task employed in the present studies makes use of similar cognitive and attentional resources, these data go some way toward an explanation of the inconsistency and variability within the line bisection literature (see Jewel \& McCourt, 2000). It appears that factors such as stimulus size and spatial location are extremely important in tasks involving horizontal size estimation. Systematic manipulation of parameters in future research could be more informative about the specificity of hemispatial bias and the distribution of attention across the visual field.

\section{AUTHOR NOTE}

Address correspondence to A. Sahraie, Vision Research Laboratories, School of Psychology, King's College, University of Aberdeen, Aberdeen AB24 2UB, Scotland (e-mail: a.sahraie@abdn.ac.uk).

\section{REFERENCES}

Anderson, B. (1996). A mathematical model of line bisection behaviour in neglect. Brain, 119, 841-850.

Barlow, H. B., \& ReEves, B. C. (1979). The versatility and absolute efficiency of detecting mirror symmetry in random dot displays. Vision Research, 19, 783-793.

Bowers, D., \& Heilman, K. M. (1980). Pseudoneglect: Effects of hemispace on a tactile line bisection task. Neuropsychologia, 18, 491-498.

Carrasco, M., Evert, D. L., Chang, I., \& Katz, S. M. (1995). The eccentricity effect: Target eccentricity affects performance on conjunction searches. Perception \& Psychophysics, 57, 1241-1261.

De Renzi, E., Gentilini, M., Faglioni, P., \& Barbieri, C. (1989). Attentional shift towards the rightmost stimuli in patients with left visual neglect. Cortex, 25, 231-237. 
DeValois, R. L., \& DeValois, K. K. (1988). Spatial vision. New York: Oxford University Press.

Drain, M., \& Reuter-Lorenz, P. A. (1996). Vertical orienting control: Evidence for attentional bias and "neglect" in the intact brain. Journal of Experimental Psychology: General, 125, 139-158.

Driver, J., \& Halligan, P. W. (1991). Can visual neglect operate in object-centred coordinates? Cognitive Neuropsychology, 8, 475-496.

Evans, C. S., Wenderoth, P., \& Cheng, K. (2000). Detection of bilateral symmetry in complex biological images. Perception, 29, 31-42.

Ferber, S., \& Karnath, H. O. (2001). Size perception in hemianopia and neglect. Brain, 124, 527-536.

Foxe, J. J., McCourt, M. E., \& JavitT, D. C. (2003). Parietal control of visuospatial attention: Line bisection judgments evaluated with high-density electrical mapping and source analysis. NeuroImage, 19, 710-726.

Gainotti, G., \& Tiacci, C. (1971). The relationships between disorders of visual perception and unilateral spatial neglect. Neuropsychologia, 9, 451-458.

Giurfa, M., Eichmann, B., \& Menzel, R. (1996). Symmetry perception in an insect. Nature, 382, 458-461.

Halligan, P. W., Manning, L., \& Marshall, J. C. (1991). Hemispheric activation versus spatio-motor cueing in visual neglect: A case study. Neuropsychologia, 29, 165-176.

Halligan, P. W., \& Marshall, J. C. (1994). Right-sided cueing can ameliorate left neglect. Neuropsychology Rehabilitation, 4, 63-73.

Heilman, K. M., Bowers, E., Valenstein, R. T., \& Watson, R. T. (1987). Hemispace and hemispatial neglect. In M. Jeannerod (Ed.), Neurophysiological and neuropsychological aspects of spatial neglect (pp. 115-150). Amsterdam: Elsevier.

Heilman, K. M., \& Van Den Abell, T. (1980). Right hemisphere dominance for attention: The mechanism underlying hemispheric asymmetries of inattention (neglect). Neurology, 30, 327-330.

Jewell, G., \& McCourt, M. E. (2000). Pseudoneglect: A review and meta-analysis of performance factors in line bisection tasks. Neuropsychologia, 38, 93-110.

Kinsbourne, M. (1970). The cerebral basis of lateral asymmetries in attention. Acta Psychologica, 33, 193-201.

KinsBouRne, M. (1987). Mechanisms of unilateral neglect. In M. Jeannerod (Ed.), Neurophysiological and neuropsychological aspects of spatial neglect (pp. 69-86). Amsterdam: Elsevier.

Kinsbourne, M. (1993). Orientational bias model of unilateral neglect: Evidence from attentional gradients within hemispace. In I. H. Robertson \& J. C. Marshall (Eds.), Unilateral neglect: Clinical and experimental studies (pp. 63-86). Hove, UK: Erlbaum.

LaBerge, D., \& Brown, V. (1989). Theory of attentional operation in shape identification. Psychological Review, 96, 101-124.

Locher, P. J., \& Wagemans, J. (1993). Effects of element type and spatial grouping on symmetry detection. Perception, 22, 565-587.

LUH, K. E. (1995). Line bisection and perceptual asymmetries in normal individuals: What you see is not what you get. Neuropsychology, 9, 435-448.

Luh, K. E., Rueckert, L. M., \& Levy, J. (1991). Perceptual asymmetries for free viewing of several types of chimeric stimuli. Brain \& Cognition, 16, 83-103.

Manning, L., Halligan, P. W., \& Marshall, J. C. (1990). Individual variation in line bisection: A study of normal subjects with application to the interpretation of visual neglect. Neuropsychologia, 28, 647-655.

Mapstone, M., Weintraub., S., Nowinski, C., Kaptanoglu, G., Gitelman, D. R., \& Mesumam, M. M. (2003). Cerebral hemispherical specialization for spatial attention: Spatial distribution of searchrelated eye fixation in the absence of neglect. Neuropsychologia, 41, 1396-1409.

Mattingley, J. B., Bradshaw, J. L., Nettleton, N. C., Bradshaw, J. A. (1994). Can task specific perceptual bias be distinguished from unilateral neglect? Neuropsychologia, 32, 805-817.

McCourt, M. E., Freeman, P., Tahmahkera-Stevens, C., \& Chaussee, M. (2001). The influence of unimanual response on pseudoneglect magnitude. Brain \& Cognition, 45, 52-63.

McCourt, M. E., \& Garlinghouse, M. (2000a). Asymmetries of visuospatial attention are modulated by viewing distance and visual field elevation: Pseudoneglect in peripersonal and extrapersonal space. Cortex, 36, 715-731.
McCourt, M. E., \& Garlinghouse, M. (2000b). Stimulus modulation of pseudoneglect: Influence of line geometry. Neuropsychologia, $\mathbf{3 8}$ 520-524

McCourt, M. E., \& Jewell, G. (1999). Visuospatial attention in line bisection: Stimulus modulation of pseudoneglect. Neuropsychologia, 37, 843-855.

McCourt, M. E., \& Olafson, C. L. (1997). Cognitive and perceptual influences on visual line bisection: Psychophysical and chronometric analyses of pseudoneglect. Neuropsychologia, 35, 369-380.

McManus, I. C., \& TomLinson, J. (2004). Objects look different sizes in the right and left eyes. Laterality, 3, 245-265.

Milner, A. D., Brechmann, M., \& Pagliarni, L. (1992). To halve or halve not: An analysis of line bisection judgments in normal subjects. Neuropsychologia, 30, 515-526.

Milner, A. D., \& Harvey, M. (1995). Distortion of size perception in visuospatial neglect. Current Biology, 5, 85-89.

Milner, A. D., Harvey, M., Roberts, R. C., \& Forster, S. V. (1993). Line bisection errors in visual neglect: Misguided action or size distortion? Neuropsychologia, 31, 39-49.

Moller, A. P., \& ThorNhill, R. (1998). Bilateral symmetry and sexual selection: A meta-analysis. American Naturalist, 151, 174-192.

Neilson, K. E., Intriligator, J., \& Barton, J. J. (1999). Spatial representation in the normal visual field: A study of hemifield line bisection. Neuropsychologia, 37, 267-277.

Nicholls, M. E. R., BradshaW, J. L., \& Mattingley, J. B. (1999). Free-viewing perceptual asymmetries for the judgment of shade, numerosity and size. Neuropsychologia, 37, 307-314.

Nicholls, M. E. R., Bradshaw, J. L., \& Mattingley, J. B. (2005). The effect of strategy on pseudoneglect for luminance judgments. Cognitive Brain Research, 25, 71-77.

Nicholls, M. E. R., Mattingley, J. B., Berberovic, N., Smith, A., \& BradshaW, J. L. (2004). An investigation of the relationship between free-viewing perceptual asymmetries for vertical and horizontal stimuli. Cognitive Brain Research, 19, 289-301.

Nicholls, M. E. R., \& Roberts, G. R. (2002). Pseudoneglect: A scanning, pre-motor or attentional bias? Cortex, 38, 113-136.

ORR, C. A., \& Nicholls, E. R. (2005). The nature and contribution of space- and object-based attentional biases to free-viewing perceptual asymmetries. Experimental Brain Research, 162, 384-393.

Post, R. B., Caufield, K. J., \& Welch, R. B. (2001). Contributions of object- and space-based mechanisms to line bisection errors. Neuropsychologia, 39, 856-864.

Rainville, S. J. M., \& Kingdom, F. A. A. (2000). The functional role of oriented spatial filters in the perception of mirror symmetrypsychophysics and modelling. Vision Research, 40, 2621-2644.

RapCSAK, S. Z., Watson, R. T., \& Heilman, K. M. (1987). Hemispace and visual-field interactions in visual extinction. Journal of Neurology, Neurosurgery, \& Psychiatry, 50, 1117-1124.

Shelton, P. A., Bowers, D., \& Heilman, K. M. (1990). Peripersonal and vertical neglect. Brain, 113, 191-205.

Steenhuis, R. E., \& Bryden, M. P. (1989). Different dimensions of hand preference that relate to skilled and unskilled activities. Cortex, 25, 285-304.

Tipper, S. P., \& Behrmann, M. (1996). Object-centered not scenebased visual neglect. Journal of Experimental Psychology: Human Perception \& Performance, 22, 1261-1278.

Tотн, C., \& KIRK, A. (1996). A normal bias toward a pictorially defined top in line bisection. Canadian Journal of Neurological Science, $\mathbf{2 3}$, $110-113$

Wenderoth, P. (1994). The salience of vertical symmetry. Perception, 23, 221-236.

Wilkinson, D. T., \& Halligan, P. W. (2002). The effects of stimulus symmetry on landmark judgments in left and right visual fields. Neuropsychologia, 40, 1045-1058.

Wilkinson, D. T., \& Halligan, P. W. (2003). Stimulus symmetry affects the bisection of figures but not lines: Evidence from eventrelated fMRI. NeuroImage, 20, 1756-1764.

Wolfe, J. M., O'Neill, P., \& Bennett, S. C. (1998). Why are there eccentricity effects in visual search? Visual and attentional hypotheses. Perception \& Psychophysics, 60, 140-156.

(Manuscript received April 5, 2006; revision accepted for publication September 12, 2006.) 